\title{
A Multipopulation PSO Based Memetic Algorithm for Permutation Flow Shop Scheduling
}

\author{
Ruochen Liu, Chenlin Ma, Wenping Ma, and Yangyang Li \\ Key Laboratory of Intelligent Perception and Image Understanding of Ministry of Education of China, Xidian University, \\ Xian 710071, China \\ Correspondence should be addressed to Ruochen Liu; aliang3399@gmail.com
}

Received 5 August 2013; Accepted 24 September 2013

Academic Editors: L. D. S. Coelho and Y. Yin

Copyright (C) 2013 Ruochen Liu et al. This is an open access article distributed under the Creative Commons Attribution License, which permits unrestricted use, distribution, and reproduction in any medium, provided the original work is properly cited.

The permutation flow shop scheduling problem (PFSSP) is part of production scheduling, which belongs to the hardest combinatorial optimization problem. In this paper, a multipopulation particle swarm optimization (PSO) based memetic algorithm (MPSOMA) is proposed in this paper. In the proposed algorithm, the whole particle swarm population is divided into three subpopulations in which each particle evolves itself by the standard PSO and then updates each subpopulation by using different local search schemes such as variable neighborhood search (VNS) and individual improvement scheme (IIS). Then, the best particle of each subpopulation is selected to construct a probabilistic model by using estimation of distribution algorithm (EDA) and three particles are sampled from the probabilistic model to update the worst individual in each subpopulation. The best particle in the entire particle swarm is used to update the global optimal solution. The proposed MPSOMA is compared with two recently proposed algorithms, namely, PSO based memetic algorithm (PSOMA) and hybrid particle swarm optimization with estimation of distribution algorithm (PSOEDA), on 29 well-known PFFSPs taken from OR-library, and the experimental results show that it is an effective approach for the PFFSP.

\section{Introduction}

As a well-known problem in the area of scheduling, PFSSP is defined as the processing sequence of all jobs is the same for all machines, for minimizing the maximum completion time (i.e., makespan) [1]. Concerning its complexity, PFSSP has been proved to be a combinatorial optimization problem and a nondeterministic polynomial-time (NP) hard combinatorial optimization problem. Nevertheless, it is important to develop effective and efficient approaches for PFSSP due to its significance in theory and practical engineering application.

During the past decades, many heuristic methods have been introduced for solving PFSSP, and they can be classified into three categories: constructive heuristic methods, improved heuristic methods, and hybrid heuristic algorithm [2]. The algorithms proposed in [3-5] belong to the constructive heuristic methods. These methods get the solutions by rules defined in advance, but the rules are not suitable for every environment. Then, some researchers made use of improved heuristic to get the solutions, including simulated annealing algorithm [6], Tabu search method [7], genetic algorithm $[8,9]$, and particle swarm optimization algorithm [10]. But these algorithms get the local optimal value at the most time and have some other limitations. However, these algorithms suffer from time-consuming and parameter dependent. Hybrid heuristic algorithms also called as memetic algorithms (MA) can be considered as a union of a population-based global search and local improvements and result in more robust and effective optimization tools. It is well known that the performances of global search algorithms such as evolutionary algorithm and PSO can be improved by combining with problem-dependent local searches. For PFSSP, many hybrid heuristic algorithms have also been proposed. In [11], simulated annealing (SA) and genetic algorithm-(GA-) based hybrid heuristic algorithms were proposed by incorporating a modified Nawaz-EnscoreHam (NEH) [12] heuristic. In [13], PSO algorithm combined with the variable neighborhood-based local search algorithm is proposed. In [2], an efficient particle swarm optimization based mimetic algorithm (MA) for PFSSP is proposed. 
In [14], a discrete particle swarm optimization (DPSO) algorithm is proposed to solve permutation flow shop scheduling problems, which use a local search procedure to escape the algorithm from the local optima. Reference [15] presented a review and comparative evaluation of heuristics and metaheuristics for the permutation flow shop problem with the makespan criterion. A comparison of 25 methods, ranging from the classical Johnson's algorithm (dispatching rules) to the recent metaheuristics, was proposed in the paper. In addition, an evaluation composed of 120 instances of different sizes was introduced as well. Reference [16] introduced a similar particle swarm optimization algorithm (SPSOA) applied for permutation flow shop scheduling to minimize makespan, which is based on the improvement of the option modes of the global optimal solution and the local optimal solution in PSO. In [17], a novel particles warm optimization (NPSO) algorithm was presented and successfully applied to permutation flow shop scheduling. In [18], a hybrid simulated annealing algorithm is proposed for the job shop scheduling problem with the objective of minimizing total weighted tardiness. The proposed algorithm was based on a novel immune mechanism whose fundamental idea was as follows: the bottleneck jobs existing in each scheduling instance generally constitute the key factors in the attempt to improve the quality of final schedules, and, thus, the sequencing of these jobs needs more intensive optimization.

In this paper, we propose a multipopulation PSO-based MA algorithm. The novelty of the algorithm lies in several aspects. First, multipopulation scheme is used to increase the diversity of the population. In the proposed algorithm, the whole population is divided into three subpopulations in which each particle evolves itself by standard PSO and then updates its best position with different local search schemes. Secondly, the cooperation among the subpopulations is achieved by an estimation of distribution algorithm (EDA) [19]. The best particle of each subpopulation is selected to construct a probabilistic model which characterizes the distribution of promising solutions in the search space in each generation. Third, to make the standard PSO suit for the PFSSP, a ranked-order value (ROV) [20] rule is presented to convert the continuous position of particles to permutation of jobs.

The rest of this paper is organized as follows. In Section 2, some related backgrounds about PFSSP and the standard PSO are introduced, respectively. In Section 3, the framework of MPSOMA algorithm is proposed, and solution representations, multipopulation cooperative coevolution, and local search schemes are introduced in detail. In Section 4, 29 wellknown PFFSPs taken from OR-library are used to evaluate the proposed algorithm. Finally, summary and conclusions are drawn in Section 5.

\section{Related Background}

2.1. PFSSP. PFSSP can be stated as follows: there are $n$ jobs to be scheduled with known processing time on $m$ machines. At one time, each job can be processed on one machine at most and each machine can process one job at most. All of the jobs should be processed with the same permutation in all machines. The objective of PFSSP is to find an appropriate permutation schedule for jobs that minimizes the maximum completion time.

Let $\pi=\left\{j_{1}, j_{2}, \ldots, j_{n}\right\}$ be a permutation of all jobs, let $p_{i, j}$ be the process time of the job $i$ on the machine $j$, and let $C\left(j_{i}, k\right)$ be the completion time of job $j_{i}$ on machine $k$; the completion time $C\left(j_{i}, k\right)$ can be calculated as follows:

$$
\begin{gathered}
C\left(j_{1}, 1\right)=p_{j_{1}, 1}, \\
C\left(j_{i}, 1\right)=C\left(j_{j-1}, 1\right)+p_{j i, 1}, \quad i=2, \ldots, n, \\
C\left(j_{1}, k\right)=C\left(j_{1}, k-1\right)+p_{j_{1}, 1}, \quad k=2, \ldots, m, \\
C\left(j_{i}, k\right)=\max \left\{C\left(j_{i-1}, k\right), C\left(j_{i}, k-1\right)\right\}+p_{j i, k}, \\
i=2, \ldots, n, \quad k=2, \ldots, m, \\
C_{\max }(\pi)=C\left(j_{n}, m\right) .
\end{gathered}
$$

The PFSSP is to find a permutation $\pi^{*}$ in the set of all permutation $\Pi$, such that

$$
C_{\max }\left(\pi^{*}\right) \leq C\left(\pi_{n}, m\right) \quad \forall \pi \in \Pi .
$$

2.2. Standard PSO Algorithm. PSO is inspired by the social interaction behavior of birds flocking and fish schooling [21]. To search for the optimal solution, each bird, which is typically called a "particle," updates its flying velocity and current position iteratively according to its own flying experience and other "particles" flying experience. At each iteration, the velocity vector for each particle is modified based on three parameters: the particle momentum, the best position reached by the particle, and that of all particles up to the current stage. Then, according to the determined velocity for each particle, the particle is moved to its next position. In PSO, a population of conceptual "particle" is initialized with random positions $X_{i}$ and velocities $V_{i}$, and a function $f$ is evaluated by using the particle's positional coordinates as input values. In an $n$-dimensional search space, $X_{i}=$ $\left(X_{i 1}, X_{i 2}, \ldots, X_{i d}\right)$ and $V_{i}=\left(V_{i 1}, V_{i 2}, \ldots, V_{i d}\right)$. Positions and velocities are adjusted, and the function is evaluated with the new coordinates at each time step. The basic update equations for the $d$ th dimension of the $i$ th particle in PSO are expressed in (7):

$$
\begin{gathered}
x_{i, j}(t+1)=x_{i, j}(t)+v_{i, j}(t+1) \quad j=1, \ldots, d, \\
v_{i, j}(t+1)=w v_{i, j}(t)_{i, j}+c_{1} r_{1}\left(p_{\text {best }, j}-x_{i, j}(t)\right) \\
+c_{2} r_{2}\left(g_{\text {best }, j}-x(t)\right) \quad j=1, \ldots, d,
\end{gathered}
$$

where $w$ is the inertia weight that controls the impact of previous velocity of particle on its current one. $c_{1}$ and $c_{2}$ are positive constant parameters called acceleration coefficients which control the maximum step size between successive iterations. $r_{1}$ and $r_{2}$ are two independently uniformly distributed random variables in range $[0,1] . P_{\text {best }}=$ $\left[p_{\text {best }, 1}, p_{\text {best }, 2}, \ldots, p_{\text {best }, d}\right]$ is the local best solution found so 
The flowchart of standard PSO

Step 1. Initialize a population of particles (viz, initial swarm);

Step 2. Evaluate the objective values of all particles in the swarm;

Step 3. If a stopping criterion is met, output $g_{\text {best }}$ and its objective value; otherwise, go to Step 4;

Step 4. Update the velocity and position of each particle by using (7) and (8);

Step 5. For each particle, compare its current objective value with the objective value of its $p_{\text {best }}$. If current value is better, then update $p_{\text {best }}$ and its objective value with the current position and objective value. Determine the best particle in the current swarm. If its objective value is better than the objective value of $g_{\text {best }}$, then update $g_{\text {best }}$. Return to Step 2 .

Algorithm 1: The flowchart of standard PSO.

Basic flowchart of MPSOMA

Step 1. $t=1$, Generate a initial population $Q$;

Step 2. Evaluate the objective values of all particles in $Q$;

Step 3. If $t>t_{\max }$, output the optimal sequences of job, otherwise divide $Q$ into three subpopulations $Q_{1}, Q_{2}$, and $Q_{3}$, go to Step 4;

Step 4. Evolve each subpopulation by adopting PSO and obtain three temporary subpopulations $Q_{1}^{\prime}, Q_{2}^{\prime}$, and $Q_{3}^{\prime}$;

Step 5. Optimize each subpopulation by different local search strategies. $Q_{1}^{\prime}$ and $Q_{3}^{\prime}$ are updated by using IIS local search and $Q_{2}^{\prime}$ is optimized by using VNS. Then three temporary subpopulations are denoted as $Q_{1}^{\prime \prime}, Q_{2}^{\prime \prime}$, and $Q_{3}^{\prime \prime}$;

Step 6. Evaluate the objective values of all particles in $Q_{i}^{\prime \prime}, i=1,2,3$ and choose the best particle from the three subpopulations decode them and three corresponding sequences of job $\pi_{1 \text { best }}, \pi_{2 \text { best }}, \pi_{3 \text { best }}$ are obtained; the worst particle is also selected from the three subpopulations and the three corresponding sequences of job are denoted as $\pi_{1 \text { wor }}, \pi_{2 \mathrm{wor}}, \pi_{3 \mathrm{wor}}$. Then $\pi_{1 \text { best }}, \pi_{2 \text { best }}, \pi_{3 \text { best }}$ are used to build a probabilistic model by EDA;

Step 7. Three new sequences of job $\pi_{1}, \pi_{2}, \pi_{3}$ are sampled from the probabilistic model, if the maximum completion time of $\pi_{i}$ is less than that of $\pi_{i \text { wor }}$, then replace the worst individual of each subpopulation by $\pi_{i}$, here $i=1,2,3$;

Step 8. Evaluate the objective values of all particles in three temporary subpopulations and choose the best particle from three subpopulations, decode it to sequence of job $\pi_{g_{\text {best }}}$ and SA local search is applied on $\pi_{g_{\text {best }}}$ and obtain a new sequence of job $\pi_{g_{\text {best }}^{\prime}}^{\prime}$. If the maximum completion time of $\pi_{g_{\text {best }}}^{\prime}$ is less than that of $\pi_{g_{\text {best }}}$, then use $\pi_{g_{\text {best }}^{\prime}}$ to replace $\pi_{g_{\text {best }}}$;

Step 9. Encode all sequences of jobs to the positions of particles and unite the three subpopulations into a population, $t=t+1$. Return to Step 2 .

Algorithm 2: The flowchart of MPSOMA.

far by the $i$ th particle, while $g_{\text {best }}=\left[g_{\text {best }, 1}, p_{\text {best }, 2}, \ldots, p_{\text {best }, d}\right]$ represents the positional coordinates of the fittest particle found so far in the entire community or in some neighborhood of the current particle. Once the iterations are terminated, most of the particles are expected to converge to a small radius surrounding the global optima of the search space. There are two common conditions used for terminating the iterative process. PSO stops when it exceeds the predefined number of iterations, or there is negligible change for particles in successive iterations.

Similar to genetic algorithms, PSO is also a populationbased iterative algorithm. But PSO requires less computational bookkeeping and generally fewer lines of code. Unlike the GA, PSO does not have complicated evolutionary operators such as crossover and mutation. The process of the standard PSO is shown in Algorithm 1.

\section{Multipopulation PSO-MA}

The multipopulation PSO-MA algorithm is proposed in this section; framework its described in Algorithm 2. First, generate an initial swarm and divide it into three subpopulations evenly. Particle in each subpopulation updates its velocities and positions by using (7). And then two local searches are applied to all particles of three subpopulations with different probabilities. (IIS Individual improvement scheme) local search is applied on subpopulation1 and subpopulation3, while VNS (variable neighborhood search) local search is used to update subpopulation2. The best particle of each subpopulation is chosen to construct the EDA probabilistic model. From the probabilistic model, we sample three new particles which are used to update the worst individuals of the corresponding subpopulation. Then, the best individual 
is selected from all individuals of three subpopulations to be performed (SA simulated annealing). Finally, $g_{\text {best }}$ is updated.

\subsection{Solution Representation and Population Initialization.} One of the key issues when designing the PSO algorithm is its solution representation. PFSSP is set in a discrete space. It is obvious that standard PSO equations cannot be used to generate a discrete job permutation since positions and velocities are real-valued. So, the most important issue in applying PSO to PFSSP is to develop a suitable mapping between positions of particles and job sequence.

In this paper, a ROV rule [20] based on random key representation is used to convert the continuous position $X_{i}=\left[x_{i, 1}, x_{i, 2}, \ldots, x_{i, n}\right]$ of particles in PSO to permutation of jobs $\pi=\left\{j_{1}, j_{2}, \ldots, j_{n}\right\}$; thus, the performance of the particle can be evaluated. In our ROV rule, the position information $X_{i}=\left[x_{i, 1}, x_{i, 2}, \ldots, x_{i, n}\right]$ itself does not represent a sequence, whereas the rank of each position value of a particle represents a job index so as to construct a permutation of jobs. The smallest position value of a particle is assigned rank 1. Then, the second smallest position value is assigned to rank 2 . With the same way, all the position values will be handled to convert the position information of a particle to a job permutation. We provide a simple example to illustrate the ROV rule in Table 1 . In the beginning, position is $x_{i}=$ $[0.6,2.9,1.8,3.7,2.1,0.7]$. Because $x_{i, 1}=0.6$ is the smallest position value, $x_{i, 1}$ is picked first and assigned rank value 1 ; then, $x_{i, 6}=0.7$ is picked and assigned rank value 2 . Similarly, the ROV rule assigns rank values 3 to 6 to $x_{i, 3}, x_{i, 5}, x_{i, 2}$, and $x_{i, 4}$, respectively. Thus, based on the ROV rule, the job permutation is obtained $\pi=\{1,5,3,6,4,2\}$.

In the standard PSO, each particle is initialized in a real space randomly, and an initial swarm is composed by a set of particles. In this paper, we use the same method as [2] to generate the initial swarm. A solution (i.e., permutation of jobs) is generated by using NEH heuristic [12], and the rest particles are initialized as the standard PSO; namely, they are generated with a random position values and velocities in a certain interval.

The results produced by NEH heuristic or the local search are job permutations, so they should be converted to the position values of a certain particle to perform PSO based searching. The conversation is performed using the following equation [22]:

$$
\begin{array}{r}
x_{\mathrm{NEH}, j}=x_{\min , j}+\frac{\left(x_{\max , j}-x_{\min , j}\right)\left(s_{\mathrm{NEH}, j}-1+\text { rand }\right)}{n \quad}, \\
j=1,2, \ldots, n,
\end{array}
$$

where $x_{\mathrm{NEH}, j}$ is the position value in the $j$ th dimension of the particle, $s_{\mathrm{NEH}, j}$ is the job index in the $j$ th dimension of the permutation by the NEH heuristic, $x_{\max , j}$ and $x_{\min , j}$ are the upper and lower bounds of the position value, respectively, rand denotes a random number uniformly distributed in the interval $[0,1]$, and $n$ represents the number of dimensions of a position, which is equal to the number of jobs.
TABLE 1: The example of ROV rule.

\begin{tabular}{lcccccc}
\hline Dimension $j$ & 1 & 2 & 3 & 4 & 5 & 6 \\
\hline Position value & 0.6 & 2.9 & 1.8 & 3.7 & 2.1 & 0.7 \\
Ranked-order value & 1 & 5 & 3 & 6 & 4 & 2 \\
\hline
\end{tabular}

3.2. EDA for PFSSP. In the proposed algorithm, we divide the whole population into three subpopulations. The relation between the subpopulation is not competitive but cooperative coevolution. Cooperative coevolution [23] is inspired by the ecological relationship of symbiosis where different species live together in a mutually beneficial relationship. The basic idea of cooperative coevolution is to divide and conquer: divide a large system into many modules, evolve the modules separately, and then combine them together to form the whole system. In this paper, we divide the population into three subpopulations; firstly, each of these subpopulations is evolved by using the standard PSO and then updated subpopulations are optimized by local search schemes further. Then, the information of best individual of each subpopulation is shared by EDA [24].

EDAs were firstly introduced in [19] which is a class of novel population-based evolutionary algorithms. Unlike traditional evolutionary algorithms, new solutions are sampled from EDA probabilistic model which characterizes the distribution of promising solutions in the search space at each generation. Due to its effectiveness and search ability, EDA has recently attracted much attention in the field of evolutionary computation, and it has already been applied to solve combinatorial optimization problems, including the flow shop scheduling problem.

In our proposed algorithm, we select three individuals to construct a probabilistic model. In every generation, particle in each subpopulation evolves with the standard PSO operation and then updates its best position by using two different local search schemes. The best particle of each subpopulation is chosen and then decoded to the permutation of the job. Instead of using the conventional crossover and mutation operations in evolutionary algorithm, EDA estimates a probabilistic model from the information of the selected three individuals in the current generation, which is represented with a conditional probability distribution for each decision variable. Here, we will introduce the process simply.

Let three sequences of jobs corresponding to three best particles selected from three subpopulations after the local search be $\pi_{1 \text { best }}, \pi_{2 \text { best }}$, and $\pi_{3 \text { best }}$, let $\eta_{j k}$ be the number of times of appearance of job $j$ before or in the position $k$ in the subset of the selected sequences augmented by a given constant $\delta_{1}$, and let $\mu_{j[k-1]}$ be the number of times of appearance of job $j$ immediately after the job in the position $k-1$ in the subset of the selected sequences augmented by a given constant $\delta_{2}$. Here, The value of $\eta_{j k}$ refers to the importance of the order of the jobs in the sequence and $\mu_{j[k-1]}$ refers to the importance of the similar blocks of jobs in the sequences. 


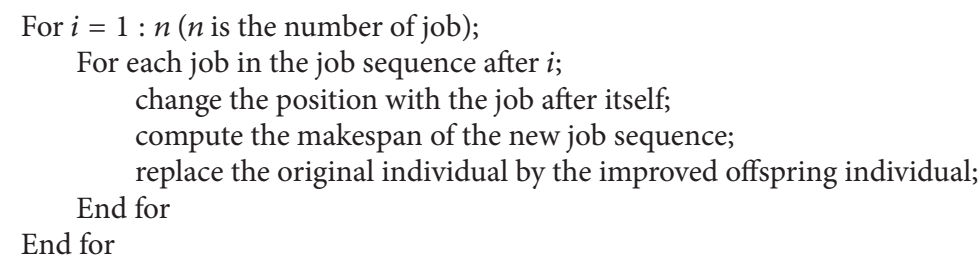

Algorithm 3: The basic flowchart of IIS local search.

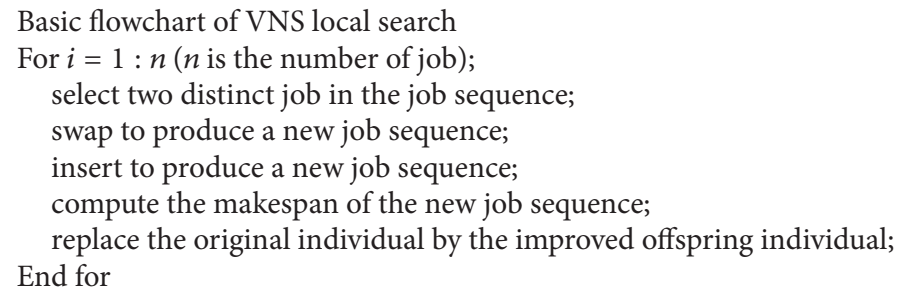

Algorithm 4: The basic flowchart of IIS local search.

We define $\rho(k, j)$ the probability of selection of the job $j$ in the $k$ th position by the following formula:

$$
\rho(k, j)=\frac{\eta_{j k} \times \mu_{j[k-1]}}{\sum_{l \in \Omega_{k}}\left(\eta_{l k} \times \mu_{l[k-1]}\right)},
$$

where $\Omega_{k}$ is the set of jobs not already scheduled until position $k$. According to this probability, for each position $k$, we select a job $j$ from the set of not already scheduled jobs in the sequence of a new individual.

In the proposed algorithm, three new sequences of jobs $\pi_{1}, \pi_{2}$, and $\pi_{3}$ are sampled from the probabilistic model. We compare $\pi_{1}, \pi_{2}$, and $\pi_{3}$ with the corresponding three worst individuals $\pi_{1 \text { wor }}, \pi_{2 \text { wor }}$, and $\pi_{3 \text { wor }}$ in the current swarm. If $\pi_{i}$ is better than $\pi_{i \text { wor }}$ and $\pi_{i}$ is unique if compared with those individuals in the current subpopulation, then, $\pi_{i \text { wor }}$ is removed from the swarm and replaced with $\pi_{i}$.

3.3. IIS Local Search. In this section, a local search named individual improvement scheme (IIS) [25] is introduced. It is performed on each corresponding sequence of the job decoded by each particle in subpopulationl and subpopulation3. The procedure of IIS local search is shown in Algorithm 3.

IIS examines each possible pairwise interchange of the job in first position, with the jobs in all other positions. Similarly, the jobs in the second and other subsequent positions are examined. Whenever there is an improvement in the objective function, the new individual is accepted. IIS local search can be viewed as a detailed neighborhood searching process, which can improve the individuals with a certain probability.

3.4. VNS Local Search. variable neighborhood search [26], a metaheuristic proposed just a few years ago, is based upon a simple principle: systematic change of neighborhood within the search. It combines efficient local optimization procedures with the heuristics which can escape from the local optima by restructuring the neighborhood. Different neighborhood provide different candidate solutions; thus, it is possible to find out a better solution.

In this paper, the particles are decoded to sequences of jobs of subpopulation2. The VNS local search is applied to all job sequences with a certain probability. In VNS, two structures of neighborhood are adopted which are called swap and insert.

Swap. Select two distinct elements from a job permutation and swap them.

Insert. Choose two distinct elements from a job permutation randomly and insert the back one before the front one.

The procedure of VNS is shown in Algorithm 4. The method described above is applied to all the particles of subpopulation 3 with probability at each generation.

The first operation leads to all possible swaps of pairs of job's positions, within all parts of solutions. If the swap moves are performed, the second structure of neighborhood consists of all possible insert moves of pairs of positions of jobs, within all parts of the so obtained solution. Then, we return to the swap with the improved solution as a current solution. We reapply this procedure until the stopping condition.

3.5. SA Local Search. SA is a neighborhood search technique which can produce good results for combinatorial problems. A standard SA procedure begins by generating an initial solution at random. At each stage, the new solution taken from the neighborhood of the current solution is accepted as the new current solution if it has a lower or equal cost; if it has a higher cost, it is accepted with a probability.

In this paper, a SA-based local search with multiple different neighborhoods is developed to enrich the local 


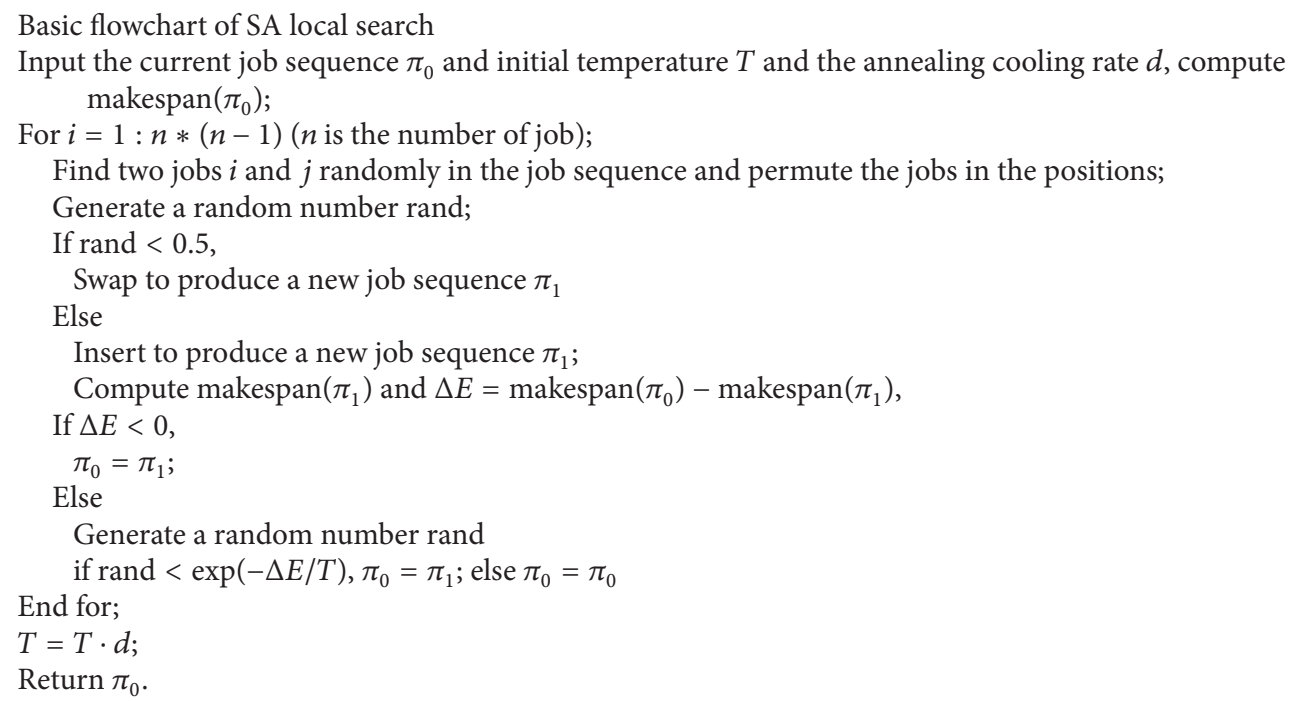

Algorithm 5: The basic flowchart of SA local search.

searching behaviors and avoid premature convergence. In SA, two different kinds of neighborhood are used. The SA algorithm randomly generates a new state in the neighborhood of the original one, which causes a change of $\Delta E$ in the objective function value. For minimization problems, the new state is accepted with probability $\min \{1, \exp (-\Delta E / T)\}$, where $T$ is a control parameter. SA provides a mechanism to probabilistically escape from local optima, and the search process can be controlled by the cooling schedule. The SAbased local search is only applied to $g_{\text {best }}$. Algorithm 5 shows the process of SA.

\section{Experimental Results}

In this section, 29 well-studied benchmarks problems which were contributed to the OR-library are selected to evaluate the performance of the proposed algorithm. The first eight problems are carl, car2, through car8 provided by Carlier [27]. The other 18 problems are called Rec01-Rec 41 given by Reeves [8]. So far, these problems have been used as benchmarks for study with different methods by many researchers.

The following parameters are used in algorithm: the size of swarm $p s=3 * n$ ( $n$ is the number of the jobs); $w=1.0, c_{1}=$ $c_{2}=2.0, x_{\min }=-4.0, x_{\max }=4.0, v_{\text {min }}=-4.0$, and $v_{\max }=4.0$; the probability of IIS local search: $P_{\text {LIS }}=0.5$; the probability of VNS local search: $P_{\mathrm{VNS}}=0.2$; the initial temperature $T_{0}=3$; the annealing cooling rate $d=0.9$; and the maximum generation is 150. Each instance is independently run 20 times for every algorithm for comparison. All parameters in the proposed algorithm are set as the compared algorithms in order to make a fair comparison; only the size of swarm is set differently from the compared algorithms since three subpopulations are employed in the MPSOMA.

In Sections 4.1 and 4.2, we will compare the performance of the proposed algorithm with those of two state-of-art
PSO algorithms PSOMA [2] and PSOEDA [22]. Then, the usefulness of local search will be discussed in Section 4.3.

The proposed algorithm is implemented in MATLAB 7.0 on a personal computer with 2.4-GHz Inter Core2 Duo CPU and 2-GB RAM and MATLAB 7.0 programming tools.

4.1. Comparisons of PSOMA and MPSOMA. To evaluate the effectiveness of multipopulation scheme in the proposed algorithm, we first compare the proposed algorithm with an effective PSO-based memetic algorithm (PSOMA) proposed by Bo Liu et al. [2]. In the PSOMA, both PSO-based searching operators and some special local searching operators are designed to balance the exploration and exploitation. SAbased local search with multiple different neighborhoods is also designed and incorporated to enrich the searching behaviors and to avoid premature convergence, and an effective adaptive meta-Lamarckian learning strategy is employed to decide which neighborhood to be used. In addition, a pairwise based local search is applied after the SA-based local search to further enhance the exploitation ability.

The experimental results are listed in Table 2. The results of PSOMA come from [2] directly. In Table 2, $C$ is the optimal makespan or lower bound value known so far, BRE represents the best relative error to $C$, ARE denotes the average relative error to $C$, WRE represents the worst relative error to $C$, and $T_{\text {avg }}$ is the average running time (in second) of 20 independent runs. The boldfaces represent the better results in Table 2.

From Table 2, it is easy to see that the proposed algorithm obtained better solutions than those obtained by PSOMA for most of test problems considered (that is to say, the $B R E$ values are very small). For those simple problems with smaller scales such as Carl Car8, the proposed algorithm can obtain the global optimal value in 20 runs. For Car5 and Car6, the proposed algorithm can provide better average relative 
TABLE 2: Comparisons of PSOMA and MPSOMA.

\begin{tabular}{|c|c|c|c|c|c|c|c|c|c|c|}
\hline \multirow{2}{*}{ Problem } & \multirow{2}{*}{$n, m$} & \multirow{2}{*}{$C$} & \multicolumn{4}{|c|}{ PSOMA } & \multicolumn{4}{|c|}{ MPSOMA } \\
\hline & & & $\mathrm{BRE}$ & $\mathrm{ARE}$ & WRE & $T_{\mathrm{avg}}(\mathrm{s})$ & $\mathrm{BRE}$ & ARE & WRE & $T_{\mathrm{avg}}(\mathrm{s})$ \\
\hline Car1 & 11,5 & 7038 & 0 & 0 & 0 & 0.68 & 0 & 0 & 0 & 24.35 \\
\hline Car2 & 13,4 & 7166 & 0 & 0 & 0 & 0.95 & 0 & 0 & 0 & 40.36 \\
\hline Car3 & 12,5 & 7312 & 0 & 0 & 0 & 1.06 & 0 & 0 & 0 & 32.31 \\
\hline Car4 & 14,4 & 8003 & 0 & 0 & 0 & 1.22 & 0 & 0 & 0 & 53.13 \\
\hline Car5 & 10,6 & 7720 & 0 & 0.018 & 0.375 & 0.70 & 0 & 0 & 0 & 22.10 \\
\hline Car6 & 8,9 & 8505 & 0 & 0.114 & 0.764 & 0.49 & 0 & $\mathbf{0}$ & $\mathbf{0}$ & 17.52 \\
\hline Car7 & 7,7 & 6590 & 0 & 0 & 0 & 0.30 & 0 & 0 & 0 & 8.09 \\
\hline Car8 & 8,8 & 8366 & 0 & 0 & 0 & 0.42 & 0 & $\mathbf{0}$ & 0 & 11.47 \\
\hline $\operatorname{Rec} 01$ & 20,5 & 1247 & 0 & 0.144 & 0.160 & 2.60 & 0 & 0 & 0 & 240.91 \\
\hline Rec03 & 20,5 & 1109 & 0 & 0.189 & 0.721 & 2.50 & 0 & 0 & 0 & 231.94 \\
\hline Rec05 & 20,5 & 1242 & 0.242 & 0.242 & 0.242 & 2.39 & 0.242 & 0.242 & 0.242 & 216.64 \\
\hline $\operatorname{Rec} 07$ & 20,10 & 1566 & 0 & 0.986 & 1.149 & 2.81 & 0 & 0 & 0 & 337.90 \\
\hline Rec09 & 20,10 & 1537 & 0 & 0.691 & 1.041 & 4.23 & 0 & 0 & 0 & 329.79 \\
\hline Rec11 & 20,10 & 1431 & 0 & 0.129 & 0.978 & 3.79 & 0 & $\mathbf{0}$ & 0 & 329.21 \\
\hline $\operatorname{Recl3}$ & 20,15 & 1930 & 0.259 & 0.893 & 1.502 & 4.64 & 0.104 & 0.891 & 1.192 & 440.11 \\
\hline Rec15 & 20,15 & 1950 & 0.051 & 0.628 & 1.076 & 5.23 & 0.615 & 0.877 & 1.333 & 455.37 \\
\hline Recl7 & 20,15 & 1902 & 0 & 1.330 & 2.155 & 4.67 & 1.419 & 1.719 & 2.103 & 447.49 \\
\hline Rec19 & 30,10 & 2093 & 0.43 & 1.313 & 2.102 & 10.49 & 0.287 & 0.506 & 0.860 & $1.66 e+03$ \\
\hline $\operatorname{Rec} 21$ & 30,10 & 2017 & 1.438 & 1.596 & 1.636 & 8.41 & 0.992 & 1.393 & 1.438 & $1.69 e+03$ \\
\hline $\operatorname{Rec} 23$ & 30,10 & 2011 & 0.596 & 1.310 & 2.038 & 9.36 & 0.398 & 0.552 & 0.796 & $1.66 e+03$ \\
\hline $\operatorname{Rec} 25$ & 30,15 & 2513 & 0.835 & 2.085 & 3.223 & 12.64 & 0.279 & 1.337 & 1.989 & $2.46 e+03$ \\
\hline $\operatorname{Rec} 27$ & 30,15 & 2373 & 1.348 & 1.605 & 2.402 & 12.15 & 0.969 & 1.336 & 1.728 & $2.25 e+03$ \\
\hline $\operatorname{Rec} 29$ & 30,15 & 2287 & 1.442 & 1.888 & 2.492 & 11.31 & 0.235 & 0.969 & 1.547 & $2.17 e+03$ \\
\hline $\operatorname{Rec} 31$ & 50,10 & 3045 & 1.510 & 2.254 & 2.692 & 37.15 & 0.699 & 1.237 & 1.792 & $1.55 e+04$ \\
\hline Rec33 & 50,10 & 3114 & 0 & 0.645 & 0.835 & 36.07 & 0 & 0.326 & 0.835 & $1.47 e+04$ \\
\hline Rec35 & 50,10 & 3277 & 0 & 0 & 0 & 29.92 & 0 & 0 & 0 & $1.32 e+04$ \\
\hline Rec37 & 75,20 & 3045 & 2.101 & 3.537 & 4.039 & 170.2 & 1.696 & 2.450 & 3.827 & $1.98 e+05$ \\
\hline Rec39 & 75,20 & 3114 & 1.553 & 2.426 & 2.830 & 155.7 & 0.581 & 1.298 & 1.792 & $1.67 e+05$ \\
\hline $\operatorname{Rec} 41$ & 75,20 & 3277 & 2.641 & 3.684 & 4.052 & 164.3 & 1.890 & 2.428 & 2.282 & $1.82 e+05$ \\
\hline
\end{tabular}

error and worst relative error than those obtained by PSOMA. For test problem Rec01 Rec11, the proposed algorithm is better than PSOMA for obtaining smaller ARE and WRE except Rec05, and the two algorithms obtained the same BRE. For test problems with 20 jobs and 15 machines, such as Rec13 and Rec17, the results of PSOMA were better than those of MPSOMA, while for the same type of test problem Rec15, BRE, ARE, and WRE values obtained by MPSOMA are better than those obtained by PSOMA. For Rec19 Rec21 with 30 jobs and 10 machines, the proposed algorithm perform better than PSOMA whatever BRE, ARE, and WRE are. For the rest of the test problems with larger scale such as Rec25 Rec41, the proposed algorithm obtained better results or similar ones as to those obtained by PSOMA. However, we also see from Table 2 that the proposed algorithm took much running time than that of PSOMA; the reason lies in three local search mechanisms which were performed on each individual in the current swarm.

4.2. Comparisons of PSOEDA and MPSOMA. To give a further evaluation on the performance of our proposed algorithm, we compare the proposed algorithm with a hybrid particle swarm optimization with estimation of distribution algorithm (PSOEDA) [22], which is recently proposed algorithm for PFSSP. PSOEDA is based on the discrete PSO and a new information sharing mechanism in swarm is proposed which not only comprise the experience of the global best solution and of the personal best solutions, but also of the information from the collective experience of all the particles. The new information sharing mechanism is converted from the EDA [24]. Some local search algorithms are also proposed to enhance the solution. All results of PSOEDA are also selected from [22]. The experiments results are shown in Table 3. Reference [22] did not provide the average running time, so we did not compare the average running time of the two algorithms in Table 3.

From Table 3, we can see that for test problems Carl Car8, PSOEDA and MPSOMA presented the same performance in 20 independent runs. For Rec01 and Rec03, the proposed algorithm is better than PSOEDA on ARE and WRE, and both algorithms obtained the same results on Rec05. For the test problems Rec07 Rec11 with 20 jobs and 
TABLE 3: Comparisons of PSOEDA and MPSOMA.

\begin{tabular}{|c|c|c|c|c|c|c|c|c|}
\hline \multirow{2}{*}{ Problem } & \multirow{2}{*}{$n, m$} & \multirow{2}{*}{ C } & \multicolumn{3}{|c|}{ PSOEDA } & \multicolumn{3}{|c|}{ MPSOMA } \\
\hline & & & BRE & ARE & WRE & BRE & ARE & WRE \\
\hline Car1 & 11,5 & 7038 & 0 & 0 & 0 & 0 & 0 & 0 \\
\hline Car2 & 13,4 & 7166 & 0 & 0 & 0 & 0 & 0 & 0 \\
\hline Car3 & 12,5 & 7312 & 0 & 0 & 0 & 0 & 0 & 0 \\
\hline Car4 & 14,4 & 8003 & 0 & 0 & 0 & 0 & 0 & 0 \\
\hline Car5 & 10,6 & 7720 & 0 & 0 & 0 & 0 & 0 & 0 \\
\hline Car6 & 8,9 & 8505 & 0 & 0 & 0 & 0 & 0 & 0 \\
\hline Car7 & 7,7 & 6590 & 0 & 0 & 0 & 0 & 0 & 0 \\
\hline Car8 & 8,8 & 8366 & 0 & 0 & 0 & 0 & 0 & 0 \\
\hline Rec01 & 20,5 & 1247 & 0 & 0.096 & 0.160 & 0 & 0 & 0 \\
\hline Rec03 & 20,5 & 1109 & 0 & 0.036 & 0.180 & 0 & 0 & 0 \\
\hline Rec05 & 20,5 & 1242 & 0.242 & 0.242 & 0.242 & 0.242 & 0.242 & 0.242 \\
\hline Rec07 & 20,10 & 1566 & 0 & 0 & 0 & 0 & 0 & 0 \\
\hline Rec09 & 20,10 & 1537 & 0 & 0.202 & 1.041 & 0 & 0 & 0 \\
\hline Recll & 20,10 & 1431 & 0 & 0.126 & 0.629 & 0 & 0 & 0 \\
\hline Rec13 & 20,15 & 1930 & 0.104 & 0.223 & 0.415 & 0.104 & 0.891 & 1.192 \\
\hline Rec15 & 20,15 & 1950 & $\mathbf{0}$ & 0.303 & 0.667 & 0.615 & 0.877 & 1.333 \\
\hline Rec17 & 20,15 & 1902 & 0 & 0.289 & 0.999 & 1.419 & 1.719 & 2.103 \\
\hline Rec19 & 30,10 & 2093 & 0.287 & 0.612 & 1.003 & 0.287 & 0.506 & 0.860 \\
\hline $\operatorname{Rec} 21$ & 30,10 & 2017 & 1.140 & 1.408 & 1.438 & 0.992 & 1.393 & 1.438 \\
\hline $\operatorname{Rec} 23$ & 30,10 & 2011 & 0.398 & 0.597 & 1.840 & 0.398 & 0.552 & 0.796 \\
\hline $\operatorname{Rec} 25$ & 30,15 & 2513 & 0.279 & 1.894 & 2.507 & 0.279 & 1.337 & 1.989 \\
\hline $\operatorname{Rec} 27$ & 30,15 & 2373 & 0.969 & 1.584 & 2.507 & 0.969 & 1.336 & 1.728 \\
\hline $\operatorname{Rec} 29$ & 30,15 & 2287 & 0.350 & 1.045 & 1.618 & 0.235 & 0.969 & 1.547 \\
\hline $\operatorname{Rec} 31$ & 50,10 & 3045 & 0.263 & 0.430 & 0.657 & 0.699 & 1.237 & 1.792 \\
\hline $\operatorname{Rec} 33$ & 50,10 & 3114 & 0 & 0.469 & 0.835 & 0 & 0.326 & 0.835 \\
\hline Rec35 & 50,10 & 3277 & 0 & 0 & 0 & 0 & 0 & 0 \\
\hline $\operatorname{Rec} 37$ & 75,20 & 3045 & 1.838 & 2.725 & 4.04 & 1.696 & 2.450 & 3.827 \\
\hline Rec39 & 75,20 & 3114 & 0.924 & 1.409 & 1.730 & 0.581 & 1.298 & 1.792 \\
\hline $\operatorname{Rec} 41$ & 75,20 & 3277 & 1.815 & 2.506 & 2.940 & 1.890 & 2.428 & 2.282 \\
\hline
\end{tabular}

10 machines, the performance of the proposed algorithm is better than that of PSOEDA and provides a better BRE and WRE. However, for the test problems Rec13 Rec17, PSOEDA can obtain better results than MPSOMA. For Rec19 Rec29 with 30 jobs, the proposed algorithm can obtain better or same results as those of PSOEDA. For the test problems with large scale, the proposed algorithm provided competitive result.

4.3. The Results of Wilcoxon Matched-Pairs Signed-Rank Test. In order to illustrate the difference between the proposed algorithm and other contrastive algorithms such as PSOMA and PSOEDA, we use the Wilcoxon matched-pairs signedrank test [28] on the optimal makespan results obtained by 20 independent runs for several test problems. The Wilcoxon Matched-Pairs Ranks test is a nonparametric test that is often regarded as being similar to a matched pair t-test and it is used to determine differences between groups of paired data when the data do not meet the rigor associated with a parametric test. The statistic results are provided in Table 4.
For Car7, Car8, Rec03, and Rec05, there is no difference among MPSOMA, PSOMA, and PSOEDA on the optimal makespan results, which means the proposed algorithm presented same results as to those of the other two algorithms on simple test problems. For Rec07 Rec 25 with 20 jobs, the differences between MPSOMA and PSOMA are significant with $P<0.05$; for the rest of test problems with 30 jobs, the differences between MPSOMA and PSOMA are still significant. For MPSOMA and PSODEA, the differences are significant for most of test problems except Rec15, Rec17, and Rec23. The results of the three test problems obtained by comparing MPSOMA and PSODEA cannot reject $H_{0}$; that is to say, there are no differences between them. The results of statistical tests confirm that MPSOMA is the best one among all the tested algorithms for most of test problems considered in this study.

4.4. The Usefulness of Different Local Search Schemes Employed in MPSOMA. In order to illustrate the usefulness of three local search such as SA, IIS, and VNS used in the proposed 
TABLE 4: The results of Wilcoxon matched-pairs signed-rank test of MPSOMA with PSOMA and PSOEDA.

\begin{tabular}{|c|c|c|c|c|}
\hline Problem & $n, m$ & $C$ & v.s. PSOMA & v.s. PSOEDA \\
\hline Car6 & 8,9 & 8505 & -2.1180 & -1.1010 \\
\hline Car7 & 7,7 & 6590 & -1.3127 & -1.2423 \\
\hline Car8 & 8,8 & 8366 & -1.3086 & -1.2126 \\
\hline $\operatorname{Rec} 03$ & 20,5 & 1109 & -1.1461 & -1.1461 \\
\hline Rec05 & 20,5 & 1242 & 1.5000 & -1.129 \\
\hline $\operatorname{Rec} 07$ & 20,10 & 1566 & -1.9852 & -1.2252 \\
\hline Rec09 & 20,10 & 1537 & -2.7304 & -2.6731 \\
\hline Recll & 20,10 & 1431 & -3.4105 & -3.0886 \\
\hline $\operatorname{Rec13}$ & 20,15 & 1930 & -3.9106 & -2.5051 \\
\hline Rec15 & 20,15 & 1950 & -2.4497 & -1.6440 \\
\hline Recl7 & 20,15 & 1902 & -3.7239 & -0.0101 \\
\hline Rec19 & 30,10 & 2093 & -1.9846 & -2.3642 \\
\hline $\operatorname{Rec} 21$ & 30,10 & 2017 & -1.9652 & -2.1660 \\
\hline $\operatorname{Rec} 23$ & 30,10 & 2011 & -2.2234 & -1.9051 \\
\hline $\operatorname{Rec} 25$ & 30,15 & 2513 & -3.0483 & -2.0333 \\
\hline
\end{tabular}

TABLE 5: Comparisons of MPSOMA-no-SA and MPSOMA.

\begin{tabular}{|c|c|c|c|c|c|c|c|c|c|c|}
\hline \multirow{2}{*}{ Problem } & \multirow{2}{*}{$n, m$} & \multirow{2}{*}{ C } & \multicolumn{4}{|c|}{ MPSOMA-no-SA } & \multicolumn{4}{|c|}{ MPSOMA } \\
\hline & & & $\mathrm{BRE}$ & ARE & WRE & $T_{\text {avg }}(\mathrm{s})$ & BRE & $\mathrm{ARE}$ & WRE & $T_{\mathrm{avg}}(\mathrm{s})$ \\
\hline Carl & 11,5 & 7038 & 0 & 0 & 0 & 18.60 & 0 & 0 & 0 & 24.35 \\
\hline Car2 & 13,4 & 7166 & 0 & 0 & 0 & 32.60 & 0 & 0 & 0 & 40.36 \\
\hline Car3 & 12,5 & 7312 & 0 & 0 & 0 & 25.33 & 0 & 0 & 0 & 32.31 \\
\hline Car4 & 14,4 & 8003 & 0 & 0 & 0 & 41.15 & 0 & 0 & 0 & 53.13 \\
\hline Car5 & 10,6 & 7720 & 0 & 0.017 & 0.155 & 14.80 & 0 & 0 & 0 & 22.10 \\
\hline Car6 & 8,9 & 8505 & 0 & 0 & 0 & 8.078 & 0 & 0 & 0 & 17.52 \\
\hline Car7 & 7,7 & 6590 & 0 & 0 & 0 & 8.077 & 0 & 0 & 0 & 8.09 \\
\hline Car8 & 8,8 & 8366 & 0 & 0 & 0 & 8.078 & 0 & 0 & 0 & 11.47 \\
\hline $\operatorname{Rec} 01$ & 20,5 & 1247 & 0.160 & 0.160 & 0.160 & 204.42 & 0 & $\mathbf{0}$ & 0 & 240.91 \\
\hline $\mathrm{Rec} 03$ & 20,5 & 1109 & 0 & 0.063 & 0.180 & 199.23 & 0 & $\mathbf{0}$ & 0 & 231.94 \\
\hline Rec05 & 20,5 & 1242 & 0.242 & 0.242 & 0.242 & 190.77 & 0.242 & 0.242 & 0.242 & 216.64 \\
\hline $\operatorname{Rec} 07$ & 20,10 & 1566 & 0 & 0.706 & 1.149 & 303.58 & 0 & 0 & 0 & 337.90 \\
\hline Rec09 & 20,10 & 1537 & 0 & 0.293 & 0.911 & 298.34 & 0 & 0 & 0 & 329.79 \\
\hline Recll & 20,10 & 1431 & 0 & 0.486 & 0.978 & 300.91 & 0 & $\mathbf{0}$ & 0 & 329.21 \\
\hline Rec13 & 20,15 & 1930 & 0.777 & 1.324 & 2.073 & 385.50 & 0.104 & 0.891 & 1.192 & 440.11 \\
\hline Rec15 & 20,15 & 1950 & 0.718 & 1.336 & 2.051 & 394.47 & 0.615 & 0.877 & 1.333 & 455.37 \\
\hline Rec17 & 20,15 & 1902 & 1.157 & 2.224 & 3.155 & 391.88 & 1.419 & 1.719 & 2.103 & 447.49 \\
\hline Rec19 & 30,10 & 2093 & 1.290 & 1.636 & 2.485 & $1.62 e+03$ & 0.287 & 0.506 & 0.860 & $1.66 e+03$ \\
\hline $\operatorname{Rec} 21$ & 30,10 & 2017 & 1.438 & 1.596 & 1.636 & $1.75 e+03$ & 0.992 & 1.393 & 1.438 & $1.69 e+03$ \\
\hline $\operatorname{Rec} 23$ & 30,10 & 2011 & 0.547 & 1.342 & 1.790 & $1.65 e+03$ & 0.398 & 0.552 & 0.796 & $1.66 e+03$ \\
\hline $\operatorname{Rec} 25$ & 30,15 & 2513 & 1.990 & 1.026 & 3.263 & $2.33 e+03$ & 0.279 & 1.337 & 1.989 & $2.46 e+03$ \\
\hline $\operatorname{Rec} 27$ & 30,15 & 2373 & 1.264 & 1.846 & 2.444 & $2.44 e+03$ & 0.969 & 1.336 & 1.728 & $2.25 e+03$ \\
\hline $\operatorname{Rec} 29$ & 30,15 & 2287 & 1.705 & 2.392 & 3.017 & $2.08 e+03$ & 0.235 & 0.969 & 1.547 & $2.17 e+03$ \\
\hline $\operatorname{Rec} 31$ & 50,10 & 3045 & 2.594 & 2.667 & 2.890 & $3.34 e+03$ & 0.699 & 1.237 & 1.792 & $1.55 e+04$ \\
\hline Rec33 & 50,10 & 3114 & 0.610 & 0.822 & 1.124 & $1.20 e+04$ & $\mathbf{0}$ & 0.326 & 0.835 & $1.57 e+04$ \\
\hline
\end{tabular}

algorithm, we will execute an extensive experiment on some test problems employed in this study.

Firstly, we removed the SA local search from the MPSOMA, namely, in the procedure of MPSOMA given in Algorithm 1; step 9 is skipped; we denote the algorithm without SA local search as MPSOMA-no-SA, and the performance of MPSOMA is compared with that of MPSOMA-noSA on the test problems used in Section 4.1. Each test problem performs 20 independent runs and record ARE, BRE, WRE, and $T_{\text {avg }}$. The experimental results are presented in Table 5. 
TABLE 6: Comparisons of usefulness of different local search settings.

\begin{tabular}{|c|c|c|c|c|c|c|c|c|c|c|c|c|}
\hline \multirow{2}{*}{ Problem } & \multicolumn{3}{|c|}{ Two IISs and one VNS (MPSOMA) } & \multicolumn{3}{|c|}{ Two VNSs and one IIS } & \multicolumn{3}{|c|}{ Three VNSs } & \multicolumn{3}{|c|}{ Three IISs } \\
\hline & BRE & ARE & WRE & BRE & ARE & WRE & $\mathrm{BRE}$ & ARE & WRE & BRE & ARE & WRE \\
\hline Car1 & 0 & 0 & 0 & 0 & 0 & 0 & 0 & 0 & 0 & 0 & 0 & 0 \\
\hline Car2 & 0 & 0 & 0 & 0 & 0 & 0 & 0 & 0 & 0 & 0 & 0 & 0 \\
\hline Car3 & 0 & 0 & 0 & 0 & 0 & 0 & 0 & 0 & 0 & 0 & 0 & 0 \\
\hline Car4 & 0 & 0 & 0 & 0 & 0 & 0 & 0 & 0 & 0 & 0 & 0 & 0 \\
\hline Car5 & 0 & 0 & 0 & 0 & 0 & 0 & 0 & 0 & 0 & 0 & 0 & 0 \\
\hline Car6 & 0 & 0 & 0 & 0 & 0 & 0 & 0 & 0 & 0 & 0 & 0 & 0 \\
\hline Car7 & 0 & 0 & 0 & 0 & 0 & 0 & 0 & 0 & 0 & 0 & 0 & 0 \\
\hline Car8 & 0 & 0 & 0 & 0 & 0 & 0 & 0 & 0 & 0 & 0 & 0 & 0 \\
\hline Rec01 & 0 & 0 & 0 & 0 & 0.128 & 0.160 & 0 & 0.128 & 0.160 & 0 & 0.144 & 0.160 \\
\hline Rec03 & 0 & 0 & 0 & 0 & 0 & 0 & 0 & 0 & 0 & 0 & 0.018 & 0.180 \\
\hline Rec05 & 0.242 & 0.242 & 0.242 & 0.242 & 0.242 & 0.242 & 0 & 0.217 & 0.242 & 0 & 0.230 & 0.242 \\
\hline $\operatorname{Rec} 07$ & 0 & 0 & 0 & 0 & 0.115 & 0.150 & 0 & 0 & 0 & 0 & 0.581 & 0.150 \\
\hline Rec09 & 0 & 0 & 0 & 0 & 0.020 & 0.130 & 0 & 0.016 & 0.195 & 0 & 0.5303 & 1.301 \\
\hline Rec1l & 0 & 0 & 0 & 0 & 0.090 & 0.489 & 0 & 0.035 & 0.345 & 0 & 1.013 & 2.865 \\
\hline Rec13 & 0.104 & 0.891 & 1.192 & 0.518 & 0.992 & 1.347 & 0.155 & 0.929 & 1.347 & 0.933 & 1.433 & 2.228 \\
\hline Rec15 & 0.615 & 0.877 & 1.333 & 0.664 & 0.918 & 1.336 & 0.761 & 0.892 & 1.482 & 0.701 & 0.918 & 1.641 \\
\hline Rec17 & 1.419 & 1.719 & 2.103 & 0 & 1.754 & 2.576 & 0.368 & 1.809 & 2.261 & 1.052 & 2.327 & 3.155 \\
\hline
\end{tabular}

It is easy to see that MPSOMA are better than MPSOMAno-SA on most of test problems. For those simple problems such as Carl Car8, the two algorithms can obtain the global optimal value in 20 runs except Car5. For Rec01 Rec07, MPSOMA showed a small advantage and had a better stability since it obtained better ARE. For rest of test problem, the performance of MPSOMA is superior to that of MPSOMAno-SA. From the view of average running time, MPSOMAno-SA without SA local search showed a little advantage over MPSOMA. We can conclude that SA local search is very important contribution on improving the performance of the proposed algorithm. The large running time taken by MPSOMA is mainly expended on two other local searches, IIS and VNS, since they are performed on all individuals in swarm other than the best one.

Secondly, we will explain why we used IIS on the odd subpopulations. Here, it is pointed out that three subpopulations are equal no matter which local search used in our study is performed arbitrary subpopulation. In the following, we will set such experiments: three subpopulations are updated by using the same local search (IIS or VNS) in step 4 or two subpopulations are updated by using VNS other than IIS like step 4 and the rest subpopulations is optimized by IIS. We performed on the first Carl Rec17 to show the effect of different experimental settings. Table 6 showed the statistic results under the same parameters setting as Section 4.1, in which two VNSs and one IIS indicates that there are two subpopulations that are updated by VNS and another subpopulation is adopted IIS in the procure of MPSOMA. Three VNSs and three IISs mean that three subpopulations are updated by using VNS or IIS.

From Table 6, we can see that for test problems Carl Car8, all of the three algorithms with different local search setting can obtains the global optimal value in 20 runs, since
BRE, ARE, and WRE resulted from them are all zeros. For most of the rest of test problems, the MPSOMA can provide better ARE and WRE. From this set of experimental results, we can see that MPSOMA with two IISs local search and one VNS local search showed a better performance than that by the other three experimental settings.

\section{Conclusion}

In this paper, we have attempted to enhance the performance of MA for PFSSP by introducing multipopulations cooperative coevolution and proposed a multipopulation particle swarm optimization (PSO) based memetic algorithm (MPSOMA). Each subpopulation is evolved by the global search mechanism, namely, the standard PSO, and then all particles in three subpopulations are updated by using different local search schemes with different probabilities which can produce offspring subpopulations with large distinction. After that, a multipopulation cooperative coevolution is realized by EDA and the information of the best particles from the different subpopulations is exchanged among these subpopulations so as to boost the proposed algorithm to find optimal solution for PFSSP. Experimental results on 26 benchmarks show that the proposed algorithm are competitive. However, the running time become large with the increasing number of job and the main reason lies in that local search is executed on all particles in three subpopulations. How to solve this problem is planned for our future work.

\section{Acknowledgments}

The authors would like to express their heartfelt gratitude to Lang Zhang, who helped in revising and polishing the English 
writing. This work was supported by the National Research Foundation for the Doctoral Program of Higher Education of China (20070701022), the National Natural Science Foundation of China under Grant (nos. 61373111, 61272279, 61103119 and 61203303), the Provincial Natural Science Foundation of Shaanxi of China (nos. 2010JM8030, DF0101110203 and 2010JQ8023), and the Fundamental Research Funds for the Central Universities (nos. K50511020014, K5051302084, K50510020011, K5051302049, and K5051302023), the Fund for Foreign Scholars in University Research and Teaching Programs (the 111 Project) (no. B07048), and the Program for New Century Excellent Talents in University (no. NCET-120920).

\section{References}

[1] J. Grabowski and J. Pempera, "New block properties for the permutation flow shop problem with application in tabu search," Journal of the Operational Research Society, vol. 52, no. 2, pp. 210-220, 2001.

[2] B. Liu, L. Wang, and Y.-H. Jin, "An effective PSO-based memetic algorithm for flow shop scheduling," IEEE Transactions on Systems, Man, and Cybernetics B, vol. 37, no. 1, pp. 18-27, 2007.

[3] H. G. Campbell, R. A. Dude, and M. L. Smith, "A heuristic algorithm for the n-job, m-machine sequencing problem," Management Science, vol. 16, no. 10, pp. 630-637, 1970.

[4] S. M. Johnson, "Optimal two-and three-stage production schedules with setup times included," Naval Research Logistics Quarterly, vol. 1, pp. 61-68, 1954.

[5] J. Liu and C. R. Reeves, "Constructive and composite heuristics solution of scheduling problem," European Journal of Operational Research, vol. 132, no. 2, pp. 439-452, 2001.

[6] F. A. Ogbu and D. K. Smith, "The application of the simulated annealing algorithm to the solution of the $\mathrm{n} / \mathrm{m} / \mathrm{Cmax}$ flowshop problem," Computers and Operations Research, vol. 17, no. 3, pp. 243-253, 1990.

[7] E. Taillard, "Some efficient heuristic methods for the flow shop sequencing problem," European Journal of Operational Research, vol. 47, no. 1, pp. 65-74, 1990.

[8] C. R. Reeves, "A genetic algorithm for flowshop sequencing," Computers and Operations Research, vol. 22, no. 1, pp. 5-13, 1995.

[9] O. Engin, G. Ceran, and M. K. Yilmaz, "An efficient genetic algorithm for hybrid flow shop scheduling with multiprocessor task problems," Applied Soft Computing Journal, vol. 11, no. 3, pp. 3056-3065, 2011.

[10] Z. Lian, X. Gu, and B. Jiao, "A novel particle swarm optimization algorithm for permutation flow-shop scheduling to minimize makespan," Chaos, Solitons and Fractals, vol. 35, no. 5, pp. 851861, 2008.

[11] T. Aldowaisan and A. Allahverdi, "New heuristics for no-wait flowshops to minimize makespan," Computers and Operations Research, vol. 30, no. 8, pp. 1219-1231, 2003.

[12] M. Nawaz, E. E. Enscore Jr., and I. Ham, "A heuristic algorithm for the m-machine, n-job flow-shop sequencing problem," Omega, vol. 11, no. 1, pp. 91-95, 1983.

[13] M. F. Tasgetiren, M. Sevkli, Y.-C. Liang, and G. Gencyilmaz, "Particle swarm optimization algorithm for permutation flowshop sequencing problem," in Ant Colony Optimization and Swarm Intelligence, vol. 3172 of Lecture Notes in Computer Science, pp. 382-389, 2004.
[14] K. Rameshkumar, R. K. Suresh, and K. M. Mohanasundaram, "Discrete particle swarm optimization (DPSO) algorithm for permutation flowshop scheduling to minimize makespan," in Proceedings of the 1st International Conference on Natural Computation (ICNC '05), Lecture Notes in Computer Science, pp. 572-581, August 2005.

[15] R. Ruiz and C. Maroto, "A comprehensive review and evaluation of permutation flowshop heuristics," European Journal of Operational Research, vol. 165, no. 2, pp. 479-494, 2005.

[16] Z. Lian, X. Gu, and B. Jiao, "A similar particle swarm optimization algorithm for permutation flowshop scheduling to minimize makespan," Applied Mathematics and Computation, vol. 175, no. 1, pp. 773-785, 2006.

[17] Z. Lian, X. Gu, and B. Jiao, "A novel particle swarm optimization algorithm for permutation flow-shop scheduling to minimize makespan," Chaos, Solitons and Fractals, vol. 35, no. 5, pp. 851$861,2008$.

[18] O. Engin, G. Ceran, and M. K. Yilmaz, "An efficient genetic algorithm for hybrid flow shop scheduling with multiprocessor task problems," Applied Soft Computing Journal, vol. 11, no. 3, pp. 3056-3065, 2011.

[19] H. Mühlenbein and G. Paaß, "From recombination of genes to the estimation of distribution. Binary parameters," in Proceedings of the 4th Conference on Parallel Problem Solving from Nature (PPSN '96), vol. 1411 of Lecture Notes in Computer Science, pp. 178-187, Berlin, Germany, September 1996.

[20] J. C. Bean, "Genetic algorithms and random keys for sequencing and optimization," ORSA Journal on Computing, vol. 6, no. 2, pp. 154-160, 1994.

[21] J. Kennedy and R. Eberhart, "Particle swarm optimization," in Proceedings of IEEE International Conference on Neural Networks, pp. 1942-1948, December 1995.

[22] H. Liu, L. Gao, and Q. Pan, "A hybrid particle swarm optimization with estimation of distribution algorithm for solving permutation flowshop scheduling problem," Expert Systems with Applications, vol. 38, no. 4, pp. 4348-4360, 2011.

[23] M. A. Potter and K. A. D. Jong, "A cooperative co-evolutionary approach to function optimization," in Proceedings of the 3rd Conference on Parallel Problem Solving from Nature, vol. 2, pp. 249-257, Jerusalem, Israel, October 1994.

[24] B. Jarboui, M. Eddaly, and P. Siarry, "An estimation of distribution algorithm for minimizing the total flowtime in permutation flowshop scheduling problems," Computers and Operations Research, vol. 36, no. 9, pp. 2638-2646, 2009.

[25] T. Aldowaisan and A. Allahverdi, "New heuristics for no-wait flowshops to minimize makespan," Computers and Operations Research, vol. 30, no. 8, pp. 1219-1231, 2003.

[26] P. Hansen and N. Mladenović, "Variable neighborhood search: principles and applications," European Journal of Operational Research, vol. 130, no. 3, pp. 449-467, 2001.

[27] J. Carlier, “Ordonnancements a contraintes disjonctives," Operations Research, vol. 12, no. 4, pp. 333-351, 1978.

[28] S. García, D. Molina, M. Lozano, and F. Herrera, "A study on the use of non-parametric tests for analyzing the evolutionary algorithms' behaviour: a case study on the CEC'2005 Special Session on Real Parameter Optimization," Journal of Heuristics, vol. 15, no. 6, pp. 617-644, 2009. 

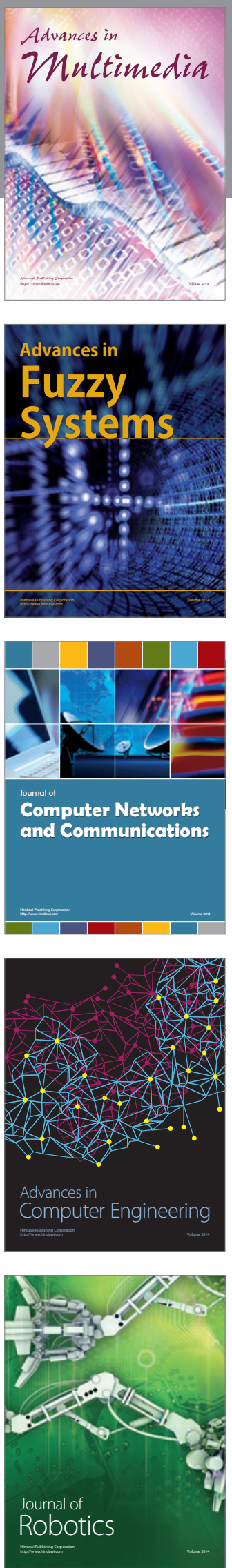

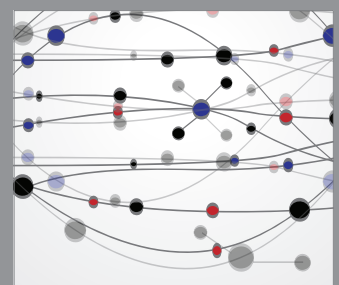

The Scientific World Journal
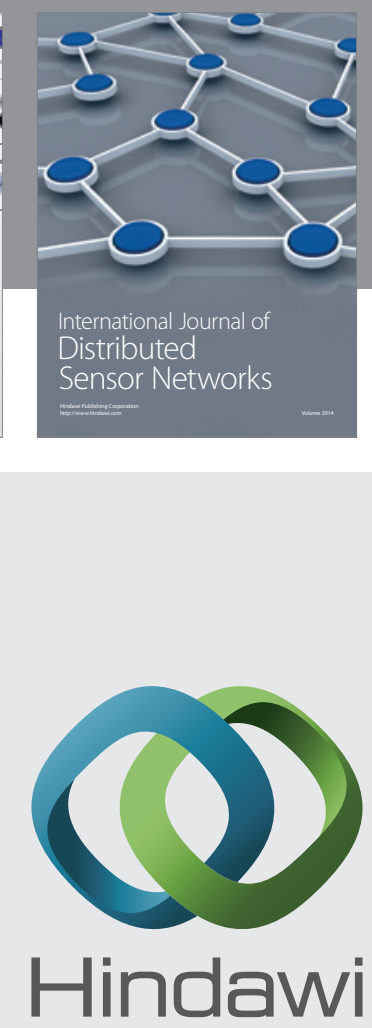

Submit your manuscripts at

http://www.hindawi.com
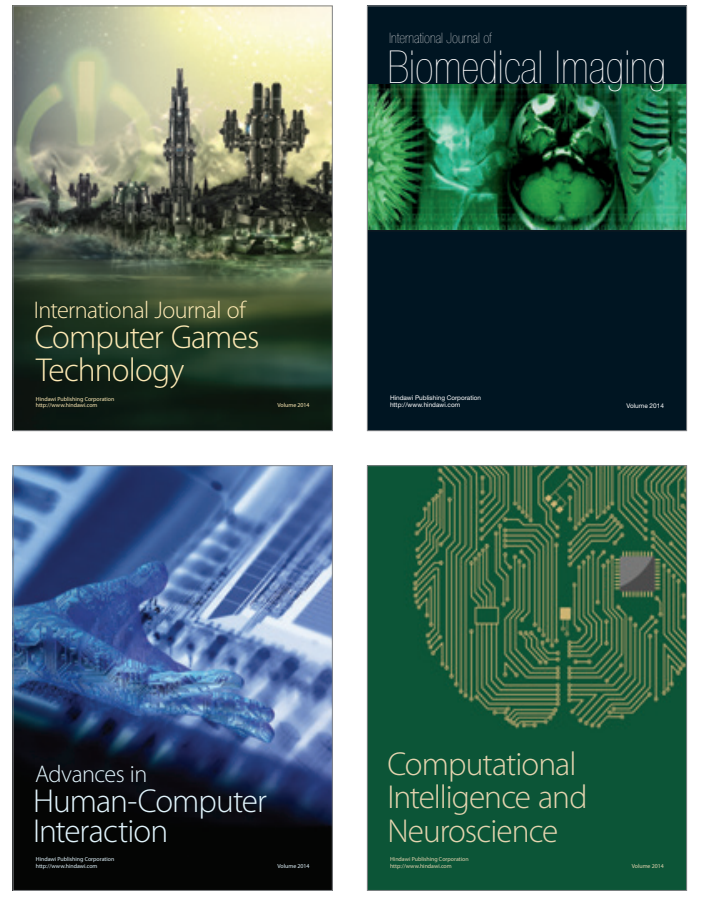
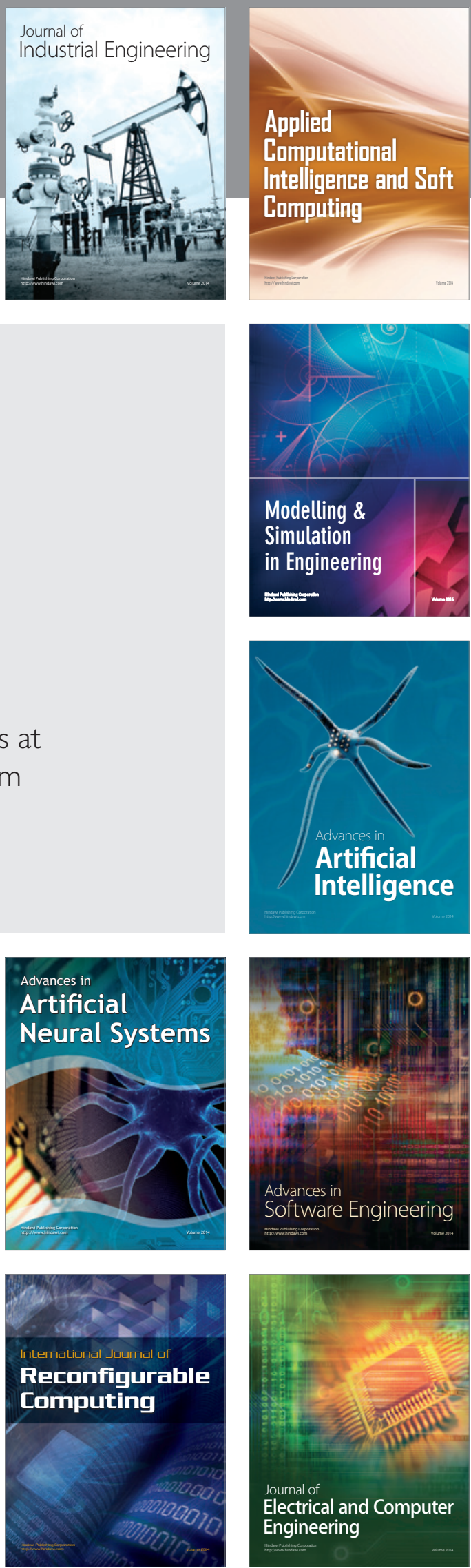